\title{
Sports Game Radial Basketball in Physical Education of Preschool Children
}

\author{
Ovchinnikov Vladimir Pavlovich ${ }^{1}$, Nesmeyanov Anatoly Alexandrovich ${ }^{2}$ and Vasilevskiy Dmitriy \\ Konstantinovich ${ }^{3}$ \\ 1. Russian State Pedagogical University, A. I. Herzen, Saint-Petersburg 190000, Russia \\ 2. President of the Federation of Piterbasket, Saint-Petersburg 190000, Russia \\ 3. Leningrad State University, Alexander Pushkin, St. Petersburg 190000, Russia
}

\begin{abstract}
The article is devoted to the application of new radial sports games basketball in physical education of preschool children. The benefits of a new game: expanding the playing space attributable to each player, limiting the opposition defenders and facilitates the use of gaming devices. The use of innovative methods in physical training of preschool children gives you the opportunity to solve successfully the whole complex of educational issues and the full development of the child.
\end{abstract}

Key words: Radial sports game basketball, preschool children, physical education, innovation of process of physical education.

\section{Introduction}

Basketball as an effective means of physical education and versatile development of working has long gained universal recognition and wide popularity. It rightly belongs among the most valuable exercise that provides physical training. Specific to this game, the highest demands on coordination have a positive impact on the development of the CNS and the formation of high activity and functional systems of behavioral reactions [1].

The first testing of this game was held in 2003 and over this relatively short period of time she acquired spread not only in Russia but also abroad (Lithuania, Poland, Sweden). Radial today basketball is becoming popular among children, youth and adult population [2].

\section{Materials and Methods}

From the classic basketball, it features the equipment used, the size and configuration of the Playground, as well as the relative simplicity of the rules of the game.

Corresponding author: Nesmeyanov Anatoly
Alexandrovich, Ph.D., professor, research fields: sports, medicine, and psychology.
If a classic basketball play strive to throw the ball into two baskets, each of which is located on the opposite sides of the rectangular area here as the goal for all players is not two, but three baskets (with shields) mounted on the strut. Assembly, installed in the center of a circle with a diameter 18 meters (Fig. 1) [3].

It is important that the game takes place on a much smaller space compared to the basketball. As a result, significantly reduced the motor load and at the same time repeatedly increases, which makes this game even more interesting and emotionally intense. At the same time, these conditions facilitate the acquisition of basic techniques and tactics of basketball, allowing the preschool children easily learn its rules and to participate in the game.

The advantage of the radial basketball is as follows: (1) the expansion of gaming space attributable to each player, limiting the opposition of the enemy and simplifies the use of gaming devices; (2) reducing the number of players, which facilitates the orientation and playing with the right solutions; (3) the Central location of the rack with three rings, widens the choice of ways of achieving the ultimate goal: throwing the ball in the basket; (4) reducing the cost of energy on the 


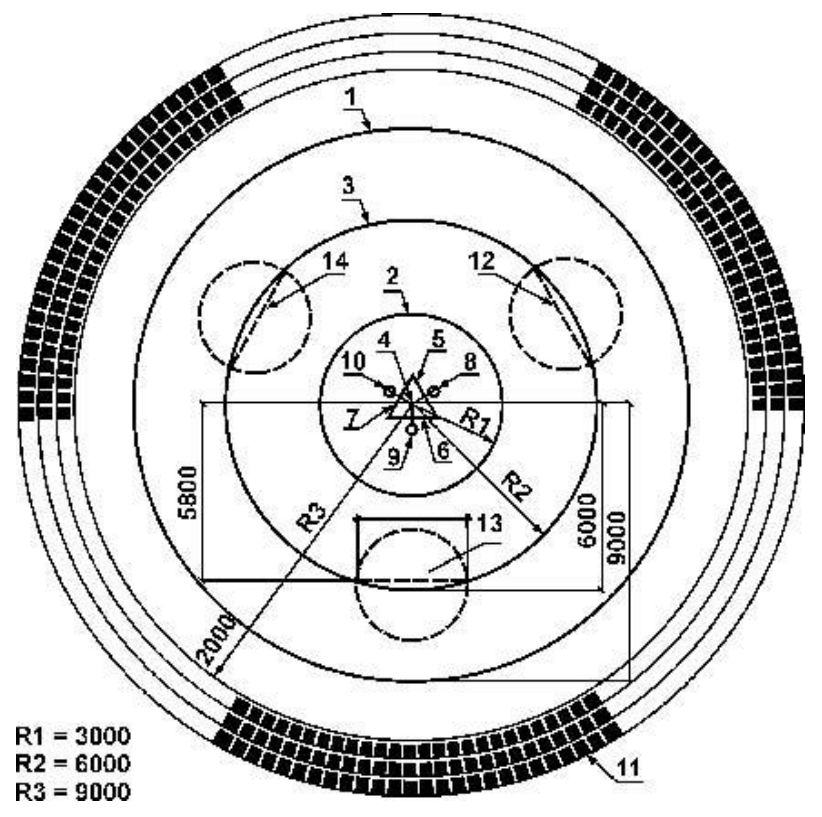

Fig. 1 The circuit court for radial basketball.

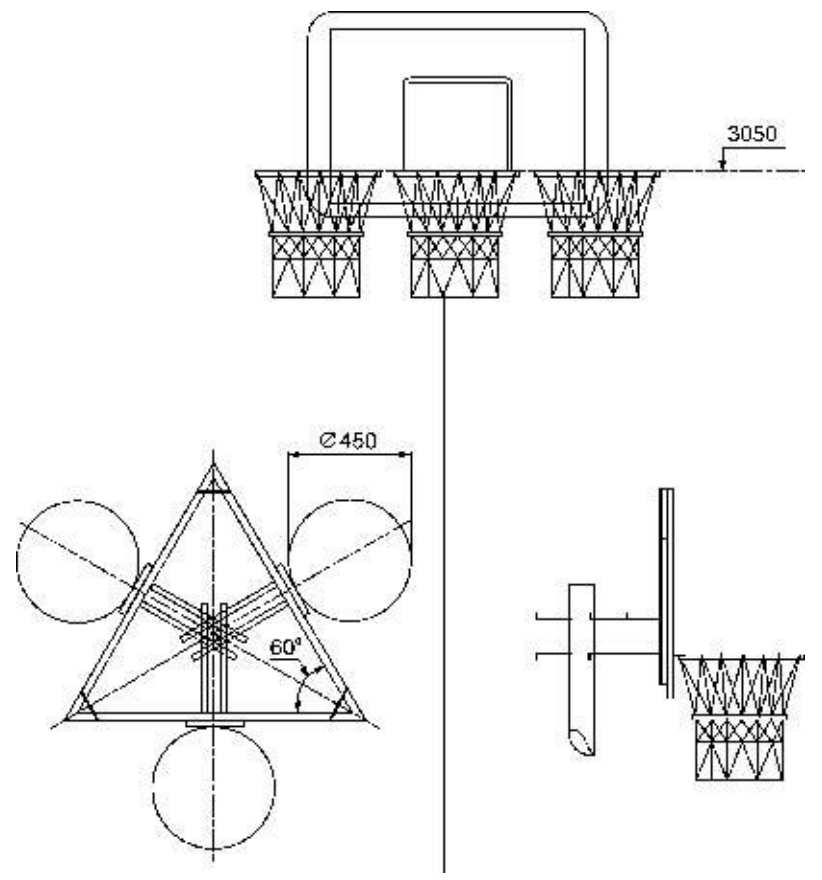

Fig. 2 Diagram of the shield with rings to play basketball in the radial.

court, in the form of a circle where the basket is installed in the center; (5) facilitating the correct choice of a place in the course of the game, where each game is located in the vicinity of one of the three baskets; (6) increase ball contact for each of the players that makes the game especially interesting for beginners.

An important feature of this version of the classic basketball is the ability to play a variety of balls and throw in the three baskets installed at any height from 1.5 to 2.5 meters. This feature not only encourages huge interest of all participants in the game, but also helps develop precise motor coordination, which is not hampered by excessive stress large muscle groups in the throwing movements of young basketball players (Fig. 2) [4].

On the accuracy of throws the ball in the basket positively affects the use of shields. It is also important that the use of three baskets at the same time, in contrast classic basketball teaches players to tactically take the right position on the court in the form of a triangle. This facilitates the transfer of the ball. Therefore, the radial basketball accelerates the formation of basic tactical interactions $(1 \times 1 ; 2 \times 2 ; 3$ $\times 3$ ), which underlie individual and group tactical actions. Considering the possibility of using the radial basketball games in physical education of preschool children, should be taken into consideration prior learning the most important elements of the art of possession. Such elements primarily include catching the ball transfer and throw the ball to the basket with two hands and dribble the ball. It is particularly important at the outset to teach children not to run with the ball in his hands and catching the ball, immediately stop, bent feet. Most often it will be games like "Who's better?", "Who exactly?", etc. Initially, there will be team games such as "Tag ball", "Wait", and "defend the fortress". After that you can include team games, but without the ball control and limiting of movement around the court (the "Firefight", "the Ball captain"). Further included is the game with ball control and shots on target located at a certain height above the ground.

\section{Conclusion}

Simplified versions of the game of basketball, which includes throwing a heavy enough ball, and in its effects on the child's body is not comparable with any other types of physical exercise. Therefore, their application in physical education of preschool children 
gives you the opportunity to solve successfully the whole complex of educational issues and the full development of the child. In itself, the familiarity with basketball meets the needs of the preschoolers and the relatively early selection of their most favorite sport is one of the most characteristic features of modern sport. The implementation of the learning game generates socially important personal qualities. Participating in team games, children learn to subordinate the interests of their own "I" the interests of the collective and accustomed to concerted action with its partners.

The rich content of the game radial basketball and relatively small costs of its implementation, allows to intensify the process of physical education in preschool educational institutions that will foster a healthy lifestyle

\section{References}

[1] Ovchinnikov, V. P. 2011. “A Device for Ball Games in
Basketball Radial 'Piterbasket' for Children of Preschool and Younger School age." In Physical Education Students, edited by Taymazov, V. A., Hook, S. C., Ashkenazi, S. M., Pshenichnikov, A. F., and Napreenko, A. A. P. Saint Petersburg: Russian State Pedagogical University, 6-77.

[2] Nesmeyanov, A. A., Nesmeyanov, D. A., Nesmeyanov, P. A., Nesmeyanov, N. A., Kasumov, A. A., Korablev, S. V., Ovchinnikov, V. P., and Cherkesov, L. Z. 2009. Apparatus for playing a game in a radial basketball piterbasket) in the period of preschool education and primary school. Patent for useful model RUS 83932.

[3] Nesmeyanov, A. A., Nesmeyanov, D. A., Nesmeyanov, N. A. Kutuzov, O. I., Tailors, I. Yu., Ovchinnikov, V. P., Nesmeyanov, N. A., Smirnov, M. B., Kazemov, A. A., and Chihladze, L. T. 2009. Site for sports games with the ball in the radial basketball "Grandpiterbasket". Patent for useful model 92802 ENG.

[4] Kazemov, A. A., Cherkesov, L. Z., Konopleva, A. N., and Nesmeyanov, A. A. 2010. "Educational Technology Education in Primary School Based on the Use of Game-Radial Basketball (Piterbasket)" Adaptive Physical Culture 2: 34-5. 